\title{
QUEEN'S
UNIVERSITY
BELFAST
}

\section{Which patients with myelofibrosis should receive ruxolitinib therapy? ELN-SIE evidence-based recommendations}

Marchetti, M., Barosi, G., Cervantes, F., Birgegård, G., Griesshammer, M., Harrison, C., Hehlmann, R., Kiladjian, J-J., Kröger, N., McMullin, M. F., Passamonti, F., Vannucchi, A., \& Barbui, T. (2016). Which patients with myelofibrosis should receive ruxolitinib therapy? ELN-SIE evidence-based recommendations. Leukemia. https://doi.org/10.1038/leu.2016.283

\section{Published in:}

Leukemia

\section{Document Version:}

Peer reviewed version

Queen's University Belfast - Research Portal:

Link to publication record in Queen's University Belfast Research Portal

\section{Publisher rights}

(c) 2016 Macmillan Publishers Limited, part of Springer Nature. All rights reserved. This work is made available online in accordance with the publisher's policies.

\section{General rights}

Copyright for the publications made accessible via the Queen's University Belfast Research Portal is retained by the author(s) and / or other copyright owners and it is a condition of accessing these publications that users recognise and abide by the legal requirements associated with these rights.

Take down policy

The Research Portal is Queen's institutional repository that provides access to Queen's research output. Every effort has been made to ensure that content in the Research Portal does not infringe any person's rights, or applicable UK laws. If you discover content in the Research Portal that you believe breaches copyright or violates any law, please contact openaccess@qub.ac.uk. 


\title{
Which patients with myelofibrosis should receive ruxolitinib therapy? ELN-SIE evidence-based recommendations
}

\author{
Marchetti Monia ${ }^{1}$, Barosi Giovanni ${ }^{2}$, Cervantes Francisco ${ }^{3}$, Birgegård Gunnar ${ }^{4}$, Griesshammer Martin ${ }^{5}$, Harrison Claire ${ }^{6}$, \\ Hehlmann Rüdiger ${ }^{7}$, Kiladjian Jean-Jacques ${ }^{8}$, Kröger Nicolaus ${ }^{9}$, McMullin Mary Frances $^{10}$, Passamonti Francesco ${ }^{11}$, \\ Vannucchi Alessandro ${ }^{12}$, Barbui Tiziano ${ }^{13}$.
}

AFFILIATIONS

${ }^{1}$ Ospedale Cardinal Massaia, Asti, Italy

${ }^{2}$ IRCCS Policlinico San Matteo Foundation, Pavia, Italy

3 Hospital Clinic, IDIBAPS, Barcelona, Spain

${ }^{4}$ Uppsala University Hospital, Uppsala, Sweden

${ }^{5}$ Johannes Wesling Klinikum Minden, Minden, Germany

${ }^{6}$ Guy's and St Thomas' NHS Foundation, London, UK

${ }^{7}$ Medizinische Fakultät Mannheim, Universität Heidelberg, Mannheim, Germany

${ }^{8}$ Centre d'Investigations Cliniques - CIC 1427, Paris, France

${ }^{9}$ University Hospital Hamburg-Eppendorf, Hamburg, Germany

${ }^{10}$ Queen's University Belfast, Belfast, UK

${ }^{11}$ University of Florence, Florence, Italy

${ }^{12}$ Ospedale di Circolo e Fondazione Macchi, Varese, Italy

${ }^{13}$ Research Foundation, Ospedale Papa Giovanni XXIII

\section{CORRESPONDING AUTHOR}

Marchetti Monia, MD, PhD Ematologia DH, SOC Oncologia, Ospedale Cardinal Massaia, Corso Dante 202, 14100 Asti, Italy Phone: +39-0141-486621 Fax: +39-0141-486026 E-mail: moniamarchettitamellini@gmail.com

RUNNING TITLE: Ruxolitinib therapy for myelofibrosis: ELN-SIE recommendations

AUTHORSHIP: Doctors Barbui and Marchetti conceived the project. All the authors contributed to the discussion on the PICOs and approved final recommendations. Doctor Marchetti conducted the critical appraisal of the literature, tracked the feedbacks from the panelists and drafted the paper. All the authors revised the manuscript and approved the final version.

\section{CONFLICT OF INTEREST STATEMENT:}

Professor Hehlmann has no competing interest for the manuscript. Doctor Barosi participated in speakers' bureau for Novartis. Doctor Kiladjian's research was funded by Novartis and AOP Orphan; he also participated in speakers' bureau for Novartis and AOP Orphan. Doctor Passamonti participated in speakers' bureau for Novartis. Doctor Griesshammer received travel reimbursement from Novartis and Shire, participated in speakers' bureaus for Baxalta, AOP Orphan, Novartis and Shire and received honoraria from Baxalta, AOP Orphan, Novartis and Shire. Doctor Vannucchi participated in advisory boards and speakers' bureaus for Novartis. Doctor Kröeger received honorarium and research funding from Novartis. Doctor McMullin received speaker fee from Novartis and Shire. Doctor Harrison received research support from Novartis and honoraria from Baxalta, Novartis, Sanofi and Shire; she joined speakers' bureaus for Baxalta, Incyte, CTI, Novartis, Sanofi and Shire. Doctor Barbui received research grants and speaker fees from Novartis. Doctor Marchetti's research was funded by Janssen, Shire and Celgene; she received travel reimbursements from Janssen, Baxalta, Celgene, Shire and consultant or speaker fees from Gilead and Celgene. Doctor Cervantes was a member of advisory boards for Novartis, Baxalta and AOP; he also joined speakers' bureaus for Novartis, Baxalta and AOP. Doctor Birgegård received research funding and speaker fees from Shire. 
Ruxolitinib is an oral JAK1/JAK2 inhibitor approved for the treatment of patients with myelofibrosis (MF) based on the results of two randomized clinical trials. However, discordant indications were provided by regulatory agencies and scientific societies for selecting the most appropriate candidates to this drug. The European LeukemiaNet and the Italian Society of Hematology shared the aim of building evidence-based recommendations for the use of ruxolitinib according to the GRADE methodology. Eighteen patientintervention-comparator-outcome profiles were listed, each of them comparing ruxolitinib to other therapies with the aim of improving one of three clinical outcomes: a) splenomegaly, b) disease-related symptoms, and c) survival. Ruxolitinib was strongly recommended for improving symptomatic or severe $(>15 \mathrm{~cm}$ below the costal margin) splenomegaly in patients with an IPSS/DIPSS risk INT2 or high. Ruxolitinib was also strongly recommended for improving systemic symptoms in patients with a MPN10 score higher than 44, refractory severe itching, unintended weight loss not attributable to other causes or unexplained fever. Because of weak evidence, the panel does not recommend ruxolitinib therapy for improving survival. Also, the recommendations given above do not necessarily apply to patients who are candidates for allogeneic stem cell transplant. 
INTRODUCTION

In the last 20 years the outcomes of blood cancers in Europe have significantly improved ${ }^{1}$ proportionally to the number of newly approved agents ${ }^{2,3}$. In 2012 two randomized phase 3 clinical trials reported outcomes for myelofibrosis (MF) patients treated with ruxolitinib, a JAK1/JAK2 inhibitor ${ }^{4,5}$. Ruxolitinib therapy was associated with reduction in splenomegaly and improvement of MF-related symptoms and, on this basis, it was rapidly approved in the US and EU. Three years later, however, the Cochrane Collaboration cast doubts on the real efficacy of this drug since a systematic literature review based on a limited follow-up concluded that ruxolitinib did not demonstrate sufficient efficacy for the two principal outcomes $^{6}$. Availability of ruxolitinib in clinical practice, prompted the British Society of Haematology ${ }^{7}$, the European Society of Medical Oncology ${ }^{8}$ and the Australian Hematology Association ${ }^{9}$ to elaborate recommendations on its use, although they were not based on an explicit GRADE approach. ${ }^{10}$ As a matter of fact, differences between marketing authorization for ruxolitinib and patient selection criteria for the COMFORT trials were reckoned as relevant hurdles by the National Institute for Clinical Excellence, which finally approved ruxolitinib in 2016 but within strict evidence-based stonemarks. ${ }^{11}$ In August 2015, the Italian Society of Haematology (SIE) and the European LeukemiaNet (ELN) shared the common effort of providing clinicians with strictly evidence-based recommendations for the selection of MF patients suitable for Ruxolitinib therapy. This paper reports the process for elaborating such statements according to the GRADE methodology and the final recommendations of the expert panel.

\section{MATERIALS AND METHODS}

A multi-country panel of 12 senior ELN members with expertise in MF management was convened. A hematologist with expertise in development of clinical practice guideline led the group through the following steps, according to the GRADE methodology ${ }^{10}$ :

1. Listing the three most relevant efficacy outcomes and the two most relevant risk outcomes

a. Efficacy outcomes: the panel chose splenomegaly, disease-related symptoms and overall survival

b. Risk outcomes: the panel chose bleeding and infection

2. Listing therapies to be compared with ruxolitinib for the achievement of each specific clinical outcome

a. Comparator therapies were hydroxycarbamide (HU) and interferon (IFN)

b. Prednisone was also considered a comparator therapy for the outcome "disease-related symptoms" 
3. Formulating an agreed definition for ambiguous terms:

a. "symptomatic" splenomegaly

b. "severe" splenomegaly

c. "relevant" disease-related symptoms

4. Listing patient-intervention-comparator-outcome (PICO) vignettes (Table 1)

5. Critical appraisal of available evidence for each of the PICOs

a. Available evidence was retrieved from the following sources: PubMed, ASH proceedings from 2013 ahead and EHA proceedings from 2013 ahead

b. Evidence was appraised according to the following hierarchy:

i. Comparative studies with appropriate directness, i.e. control arm corresponding to the comparator treatment of the PICO

ii. Comparative studies without appropriate directness, i.e. control arm does not correspond to the comparator treatment of the PICO

iii. Non-comparative studies

6. Assessing the net benefit of ruxolitinib versus the comparator treatment in each PICOs

7. Assessing the quality of evidence according to GRADE, namely based on:

a. The study design (see hierarchy above)

b. The study directness, namely the degree of similarity between the study and PICO population, intervention and outcome

8. Scoring 1 to 9 the preference of each panelist for ruxolitinib versus the comparator therapy within each PICO

9. Formulating final recommendations

10. Assessing the strength of approved recommendations, according to GRADE, namely based on the following criteria:

a. Quality of evidence

b. Benefit-to-risk balance

c. Overall uncertainty

A Delphi panel method ${ }^{12}$ was used for the steps 1, 2, 3 and 8. Final approval of definitions and recommendations was achieved informally during three meetings held in Orlando in December 2015, in Mannheim in February 2016, and in Milan in March 2016. 
63 Splenomegaly

64 Summary of evidence

65 The body of evidence supporting PICOs one to six mainly consisted of the COMFORT II trial, randomizing intermediate-2 and high risk MF patients to ruxolitinib or best-available therapy (BAT). ${ }^{4}$ The clinical outcomes of patients assigned to BAT and receiving active treatments (mostly HU) was considered a proxy for the clinical outcomes of HU-treated control patients. The COMFORT II trial reported that in patients assigned to ruxolitinib spleen volume decreased, on average, by $29 \%$ in a median of 12 weeks. ${ }^{4,13}$ The probability of maintaining a $-35 \%$ reduction in spleen volume was $48 \%$ at five years, that is a median

71 time of response of 3.2 years. ${ }^{13}$ Rather, palpable spleen size decreased for a few months and by no more 72 than $10 \mathrm{~cm}$ in a small portion of actively treated patients assigned to BAT: in these patients spleen 73 volume increased by $5 \%$ in a year. ${ }^{14}$ The efficacy of ruxolitinib onto spleen size was also supported by the 74 randomized trial COMFORT I, ${ }^{5}$ the prospective study ROBUST ${ }^{15}$ and the large phase IIIb study JUMP ${ }^{16}$. A 75 definite dose-response was reported.

76 Due to the scarce number of IFN-treated patients enrolled into the COMFORT-II control arm, evidence 77 from phase II and retrospective studies was sought. One hundred and twenty-six patients reported by 8 mainly retrospective studies were recently reviewed. ${ }^{17}$ Spleen response rates reported by the largest studies ranged from $30 \%$ to $53 \%$, and median time to response was greater than 6 months. ${ }^{17-20}$

80 Finally, we scanned evidence for patients with intermediate-1 risk disease, who were excluded from 81 enrollment into the COMFORT trials: phase $\mathrm{II}^{15}$ and phase $\mathrm{III}^{16}$ studies reported a similar efficacy of ruxolitinib onto splenomegaly in this subpopulation than in patients with intermediate-2 or high risk 83 disease.

\section{Quality of evidence}

85 The overall quality of evidence supporting the net benefit of ruxolitinib in PICOs 1 to 6 was judged to be 86 high in principle, due to the randomized design of the COMFORT II trial, but it was necessarily reduced to low or very-low due to unblindness and serious indirectness of the study. Serious indirectness was caused by a limited portion (47\%) of the control arm patients being treated with HU (the comparator therapy in PICOs 1 and 2) and by a very small portion of cytoreduction-naive patients (population of PICOs 1 to 4). The quality of evidence supporting PICOs 2 to 6 was limited by the very few patients 92 interferon efficacy in prospective or comparative studies. However, indirectness was also supported by 93 the lack of information regarding spleen size kinetics before enrollment (population of PICOs 2, 4 and 6) 
94 and of sub-analyses for patients with symptomatic splenomegaly at enrollment (population of PICOs 1,3

95 and 5). The quality of evidence was increased by a clear demonstration of a dose-response relationship 96 between ruxolitinib dose and spleen volume reduction.

97 Finally, the quality of evidence of ruxolitinib as compared with $\mathrm{HU}$ for patients with intermediate-1 risk 98 disease was judged to be very low due to the non-randomized design of the studies supporting the safety 99 and efficacy of ruxolitinib in this setting.

100 Panel discussion

101 The panel agreed that patients with symptomatic and/or severe splenomegaly not responding to prior 102 treatment should receive ruxolitinib, based on the rapid and durable reduction of spleen size reported by 103 the COMFORT trials. ${ }^{4,5,13}$ The panel deemed that cytoreduction-naïve patients with symptomatic or 104 severe splenomegaly, who also need a rapid and sustained spleen reduction, were expected to get from 105 ruxolitinib a similar incremental benefit as pre-treated patients.

106 Despite the lack of comparative evidence, the panel also recommended ruxolitinib in a limited subset of 107 patients with intermediate-1 risk disease whose quality of life is severely impaired by huge symptomatic 108 spleens or splenomegaly-related symptoms not responsive to prior therapies.

109 Finally, the panel did not provide any operative definition for "progressive splenomegaly", however, it 110 deemed that treatments aimed at preventing severe or symptomatic splenomegaly might be effectively 111 implemented in patients with progressive increase of spleen size, although no evidence from clinical 112 trials supports a specific treatment pathway.

113 Recommendations

114 Although evidence suggests that ruxolitinib is effective in reducing splenomegaly in any patient risk 115 category, the benefit/risk profile of the drug favors its use for improving splenomegaly in selected 116 patients.

117 Ruxolitinib is recommended for improving splenomegaly in:

118 - Patients with intermediate-2 or high risk disease and either symptomatic or severe splenomegaly 119 (strong recommendation)

120 - Patients with intermediate-1 risk disease and either symptomatic or severe splenomegaly not 121 responsive or intolerant to HU or interferon (weak recommendation)

122 - Patients with intermediate-1 risk disease and both symptomatic and severe splenomegaly not 123 previously treated with any cytoreductive agent (weak recommendation)

124 "Severe" splenomegaly refers to splenomegaly palpable $15 \mathrm{~cm}$ below the costal margin.

125 "Symptomatic" splenomegaly refers to the concurrent presence of a splenomegaly and local 
symptoms not attributable to other causes, such as pain in the left upper quadrant of the abdomen, or impairment of food intake due to early satiety.

128 Disease-related symptoms

129 Summary of evidence

130 Only one study provided direct evidence of ruxolitinib relative efficacy in improving disease-related 131 symptoms as compared with other therapies in patients with intermediate-2 or high- risk disease: 132 COMFORT II trial reported a similar mean improvement of EORTC Q-C30 score at week 24 in patients 133 assigned to ruxolitinib or BAT, provided that the latter were receiving an active treatment. ${ }^{14}$ Moreover, 134 no dose-response relationship was proved by any comparative or non-comparative study. Nevertheless, 135 a rapid, relevant and sustained improvement of fatigue, appetite loss and itching was consistently 136 reported with ruxolitinib treatment by the COMFORT I and ROBUST trials., ${ }^{5,15}$ Appetite loss and dyspnea, 137 on converse, significantly worsened in BAT-treated patients. ${ }^{14}$ Despite the universal use of EORTC Q-C30, 138 the questionnaire is not disease-specific and includes 30 items, therefore it cannot be feasible outside a 139 clinical trial setting. Rather, MPN10 (Table 2) is a brief disease-specific tool applied longitudinally in the 140 COMFORT-I trial and validated in several languages, showing a good feasibility. Moreover, MPN10 score 141 should be recorded in order to assess response according to IWG-MRT criteria. Despite no "clinically 142 meaningful" threshold score for MPN10 has been validated, one third of MF patients enrolled in a cross143 sectional study reported a MPN10 score higher than 44, which can be considered a good cut-off for 144 selecting patients with "relevant" disease-related symptoms, in that it corresponds to the mean value 145 plus one standard deviation. ${ }^{21}$

146 No study longitudinally assessed quality of life in patients receiving interferon or prednisone.

147 Patients with intermediate-1 risk disease enrolled into the ROBUST phase II trial achieved similar 148 symptom improvement during ruxolitinib therapy than intermediate- 2 and high risk patients. ${ }^{15}$

149 Quality of evidence

150 The overall quality of evidence was judged to be low. Despite the randomized design of the COMFORT II 151 trial, several limitations hamper its quality in supporting PICOs 7 to 9: limited size, unblindness, high rate 152 of missing data and indirectness add up with lack of a clear-cut improvement in quality of life of patients 153 assigned to ruxolitinib as compared with those assigned to active BAT. However, the consistency of the 154 data reported by COMFORT II and other studies, namely, COMFORT I and ROBUST, supports a potentially 155 relevant effect of ruxolitinib in the patients' quality of life.

156 Panel discussion 
157 The panel deemed that a systematic and quantitative assessment of MF-associated symptoms with 158 MPN10 was feasible and necessary prior to treatment decisions. The panel also considered the 159 structured summary of evidence and the poor quality of the evidence supporting a benefit of ruxolitinib 160 as compared with BAT, mainly HU. However, the rapid and sustained action of ruxolitinib upon itching, 161 appetite and fatigue was considered to be sufficient to strongly recommend it in patients carrying a high 162 burden of symptoms, that is to say, with a MPN10 score higher than 44 . The panel also deemed that 163 ruxolitinib could be recommended for controlling some specific severe symptoms, such as itching, 164 relevant weight loss or fever, irrespectively of the overall MPN10 score. The recommendation was 165 judged to be valid also in patients with intermediate-1 risk disease, while no exclusion criterion for low166 risk patients was required, since disease-related symptoms are very rare in this setting and would often 167 mean that patient risk category is increasing.

168 Recommendations

169 Accurate assessment by the tools such as MPN10 should be performed before any clinical decision 170 regarding the use of ruxolitinib for improving disease-associated symptoms.

171 Ruxolitinib is recommended for improving disease-related symptoms in patients with a MPN10 score 172 higher than 44 or refractory severe itching $($ score $>6)$ or unintended weight loss (>10\% in the last 6 173 months) not attributable to other causes or unexplained fever (Strong recommendation).

174 Survival

175 Summary of evidence

176 Search for evidence supporting PICOs 10 to 18 could retrieve only one study comparing the survival of 177 ruxolitinib-treated patients with the survival of patients assigned to other active treatments. The 178 COMFORT II trial reported a significant and relevant increase of five-year survival from 44\% (BAT) to 56\% 179 (ruxolitinib), despite cross-over, in patients with intermediate-2 or high risk disease. Spleen response 180 predicted a major improvement of survival. ${ }^{22}$ A survival benefit in favor of ruxolitinib versus other 181 therapies, consisting mainly of $\mathrm{HU}$, was also reported by two case-control studies. ${ }^{23,24}$

182 No evidence compared the overall survival of ruxolitinib-treated with interferon-treated patients.

183 No study longitudinally compared the overall survival of intermediate-1 patients receiving ruxolitinib 184 rather than other treatments.

185 Quality of evidence

186 The quality of evidence for PICOs 10 and 11 was judged to be very low despite the randomized design of 187 the COMFORT II trial, due to the limited size and lack of blindness of the study but even more due to the 
188 severe indirectness provided by the low portion of actively treated patients in the BAT arm. No 189 comparative evidence supporting a survival prolongation with ruxolitinib as compared with HU was 190 available for patients with intermediate-1 disease (PICO 12). Similarly, no evidence supported a longer 191 survival in patients treated with ruxolitinib versus interferon (PICOs 13 to 18).

192 Panel discussion

193 The panel judges that the universal prescription of a drug should be based on solid evidence supporting 194 amelioration of one of the most relevant endpoint, which is survival. The panel, therefore deemed that 195 the quality of available evidence for a survival benefit of ruxolitinib versus HU or interferon was not 196 sufficient to support a recommendation.

197 Recommendations

198 The evidence supports a survival benefit associated with ruxolitinib but its quality according to GRADE 199 was judged to be very low. Therefore, ruxolitinib should not be recommended uniquely for improving 200 survival (weak recommendation).

202 Safety: bleeding and infection

203 Comparative safety of ruxolitinib and HU or interferon was available in the COMFORT II trial: 35 out of $204146(24 \%)$ patients assigned to ruxolitinib discontinued the therapy due to adverse events, as compared 205 with only 4 out of $73(5 \%)$ patients assigned to BAT. ${ }^{13}$ Safety outcomes were not judged to 206 counterbalance the expected ruxolitinib benefit, however, the panel deemed that the reported safety 207 could be reproduced in the clinical practice only if a proactive prevention of bleeding and infection was 208 implemented.

209 Bleeding

210 Direct evidence of the relative safety of ruxolitinib versus HU was derived only from the COMFORT II 211 trial: treatment interruptions due to adverse events were more frequent in patients assigned to 212 ruxolitinib (8\% versus $3 \%$ ) as well as grade $3-4$ thrombocythopenia (15\% versus $5 \%$ ) and overall bleeding 213 events (odds ratio 2.2; 95\% Cl 1.3-2.7). ${ }^{4,13}$ Thanks to ruxolitinib dose-adjustment according to baseline 214 and follow-up platelet count, severe bleeding was rarely reported ( $2 \%$ to $3 \%$ ) either in the COMFORT 215 trials or in the JUMP study, enrolling almost only patients with baseline platelet count higher than $216100 * 10^{9} / .{ }^{4,5,13,16}$ Severe hemorrhages were also rare (less than $3 \%$ ) in studies specifically enrolling 217 patients with baseline platelet counts 50 to $100 * 10^{9} / \mathrm{I}: 5$ to $10 \mathrm{mg}$ BID ruxolitinib were administered. ${ }^{25-27}$ 218 The reported risk of bleeding related to ruxolitinib-induced thrombocytopenia prompted the panel to list 
219 the principal bleeding risk factors: Table 3 lists such factors and the panel recommends a systematic 220 assessment of these items before assigning any patient to ruxolitinib therapy. Moreover, the panel 221 suggests periodical reassessment of these factors in treated patients. Physicians are advised to ensure 222 patient awareness of his/her bleeding risk during the treatment. The panel did not provide any further 223 suggestion on starting and continuation dose, which should be titrated based on platelet count, as

224 reported by the product information.

225 Infection

226 The panel listed the most relevant issues to be considered in assessing infection risk (Table 4) and 227 deemed that ruxolitinib could not be contraindicated in any specific subset of high-infective risk patients 228 but that caution, specific monitoring or prophylactic measures are recommended in patients with at least 229 one risk factor. Screening for hepatitis viruses was deemed mandatory in order to implement monitoring 230 and/or prevention measures for potentially fatal reactivations. Specific anti-viral or anti-mycobacterial 231 preventive measures have been proposed. ${ }^{9,28-29}$ The panel recommends the infection risk to be 232 systematically assessed before administering ruxolitinib and caution in the prescription for patients 233 carrying infection risk factors (Table 4). Prophylaxis for patients at high risk of viral or mycobacterial 234 infections should be considered on a case to case basis. Moreover, physicians are advised to pursue 235 patient awareness of his/her infective risk during the treatment.

\section{Special subpopulations}

237 Due to an overall lack of direct evidence of safety and efficacy, no evidence-based recommendations

238 could be elaborated for the following specific subsets of patients.

239 Splanchnic vein thrombosis (SVT) and/or portal hypertension

240 Myelofibrosis patients with a history of SVT often have splenomegaly and also have a risk of portal 241 hypertension with risk of variceal bleeding. They were identified as a special subpopulation. The panel 242 elaborated safety recommendations based on eligibility criteria of a small phase II trial enrolling 21 243 patients with myeloproliferative neoplasms (including $12 \mathrm{MF}$ ) actively treated with anticoagulants or 244 antiplatelet drugs and both showing a platelet count higher than $100 * 10^{9} / \mathrm{I}$ at baseline and esophageal 245 varices of grade 2 or lower. ${ }^{30}$ Ruxolitinib was administered at reduced doses for patients with a baseline 246 platelet count lower than 200*10\%/I: $10 \mathrm{mg}$ BID for PV, $15 \mathrm{mg}$ BID for MF and $25 \mathrm{mg}$ BID for ET. Despite 247 the occurrence of grade 3-4 thrombocytopenia in 14.3\% of the patients, accurate dose reductions limited 248 bleeding events and only one episode of grade 2 upper gastrointestinal bleeding occurred during the 249 study period. However, the reported background rate of major hemorrhage in this setting is quite low, 
250 i.e. 3.6/100 patient-years. ${ }^{31,32}$ However, due to the large unmet needs of this patient subpopulation, the 251 panel deemed not to recommend against ruxolitinib in this setting, but to use ruxolitinib with caution 252 and to carefully titrate the dose with careful monitoring and management of portal hypertension. If 253 ruxolitinib is used in these patients, patient awareness of bleeding risk is required.

254 Hepatomegaly

255 Some splenectomized patients have been reported as achieving a reduction of hepatomegaly during 256 ruxolitinib treatment. ${ }^{33,34}$ The panel could not provide specific recommendations in favor or against the 257 use of ruxolitinib in this subset of patients. However, the panel agreed that ruxolitinib can be considered 258 in this clinical situation.

\section{Comorbidities and limited-lifespan}

260 The use of ruxolitinib was also questioned in patients with severe comorbidities which are expected to 261 limit lifespan by themselves. Only a few patients aged over 80 years were enrolled into randomized 262 COMFORT trials and the JUMP studies. ${ }^{4,5,16}$ Moreover, only $13 \%$ and $8 \%$ of patients assigned to ruxolitinib 263 and BAT, respectively, showed a performance score ECOG 2 or higher. ${ }^{4}$ Comorbidities were not 264 systematically reported by the COMFORT and the JUMP studies, but half of MF patients have a significant 265 comorbidity burden in routine care..$^{35}$ No evidence of a clear benefit-to-risk ratio of ruxolitinib as 266 compared with other available treatments has been reported in patients with limited lifespan or severe 267 comorbidities. Therefore, the panel recommended avoidance of this drug in such patients, until 268 favorable evidence is available.

269 Low-risk disease

270 The panel could not formulate any specific recommendation for the use of ruxolitinib in patients with 271 low risk disease, due to insufficient evidence.

\section{DISCUSSION}

273 Ruxolitinib represents a novel therapeutic opportunity for patients with MF. However conflicting 274 indications to its use in the clinical practice have been provided, some being based on disease risk and 275 others on symptoms. ${ }^{7-9,36,37}$ In comparison with other published statements, the ELN-SIE panel chose to 276 adopt the GRADE methodology to formulate evidence-based recommendation for an appropriate use of 277 ruxolitinib. Evidence was retrieved and appraised for 18 PICOs (Table 1) and the panel subsequently 278 formulated recommendations based on the benefit-to-risk profile of ruxolitinib, as compared with other 279 available treatments. Six evidence-based recommendations were therefore formulated suggesting to use 280 ruxolitinib for improving symptomatic or severe splenomegaly in patients with intermediate or high risk 
281 disease not responsive to cytoreductive agents. Ruxolitinib was also strongly recommended in patients 282 with relevant disease-associated symptoms, provided that symptoms were adequately quantified and 283 classified. Therefore, a strong suggestion to ruxolitinib use was formulated only for patients scoring over 28444 points by the MPN10; or suffering severe itching not responsive to standard therapy, or with either unexplained fever or unintended weight loss. Due to the urgent need for treatment, despite the scarce evidence, ruxolitinib was also recommended upfront for those patients with INT1 risk disease suffering from both symptomatic and severe splenomegaly. The panel, however, chose not to recommend ruxolitinib uniquely aimed at survival prolongation since no study has been designed and powered sufficiently to provide definite evidence. This finally suggests to target therapeutic decisions on symptoms and splenomegaly and not on survival. Such recommendations, however, also need to be timely revised according to newly coming evidence.

ELN-SIE recommendations differ from those provided by the British Committee for Standards in Hematology and by $\mathrm{ESMO}^{8}$. Both suggested ruxolitinib for patients with symptomatic splenomegaly or constitutional symptoms but did not provide the physician with a detailed support for interpreting the intensity and specificity of symptoms. ELN-SIE also struggled with using a solid methodology for evidence appraisal. The whole decision process was tracked and summarized in the paper, in order to get the best transparency and to provide the best evidence-to-recommendation adherence.

Despite the rigid GRADE methodology imposes a comparison between intervention and comparator treatments, the huge and rapid improvement of symptoms reported during ruxolitinib treatment led the panel to provide recommendations despite the scarce availability or poor quality of comparative evidence. Rather, a strict comparison-based high quality evidence was requested by the panel for considering ruxolitinib with the unique aim of improving survival. Therefore, the major result of this project was a definite distinction between the enrollment criteria of the registration trials and the decisional criteria for ruxolitinib prescription in clinical practice. Moreover, systematic and stringent definitions of "relevant" symptoms or splenomegaly were provided, favoring a homogenous and nonsubjective assignment of the most suitable patients to ruxolitinib.

Some issues were not addressed, however, by the present project, such as the rules for treatment discontinuation. IWG-MRT and ELN classified as "responsive" those patients achieving a $50 \%$ reduction of disease-related symptoms as assessed with MPN10 or a 50\% reduction of spleen size from left costal margin. ${ }^{38}$ A list of practical issues are faced in assessing the clinical response to ruxolitinib, such as appropriate timing of response assessment in patients receiving full dose or suboptimal doses. ${ }^{39}$ The panel chose not to provide specific recommendations on the modality and timing of response 
313 assessment or drug tapering before interruption. However, this was considered to be a relevant issue. 314 Furthermore, we did not include recommendations for ruxolitinib in patients who are candidates for 315 allogeneic SCT, since an EBMT/ELN consensus conference recently provided specific indications on 316 transplantation and peri-transplant therapies. ${ }^{40}$ To be definitive on the role of ruxolitinib as bridge to 317 transplant, we decided to wait results from ongoing prospective trials. However, we have to mention 318 that the vast majority of patients with indications to allogeneic SCT are on ruxolitinib treatment. Nor 319 does this paper address combination therapies including ruxolitinib, since only preliminary data are 320 available from phase $1 / 2$ studies. Finally, decision models estimated that ruxolitinib might reduce 321 disease-related costs in responders, but the overall value-for-cost of the drugs has not been completely 322 ascertained yet. ${ }^{42-43}$ Therefore, the present recommendations did not consider cost among the relevant 323 GRADE outcomes. However, the panel deemed that an appropriate clinical use of ruxolitinib should 324 assure a favorable value-for-cost. 


\section{REFERENCES}

1. Sant M, Minicozzi P, Mounier M, Anderson LA, Brenner H, Holleczek B, et al; EUROCARE-5 Working Group. Survival for haematological malignancies in Europe between 1997 and 2008 by region and age: results of EUROCARE-5, a population-based study. Lancet Oncol 2014;15:931-42.

2. Lichtenberg FR. The impact of pharmaceutical innovation on premature cancer mortality in Switzerland, 19952012. Eur J Health Econ (in press)

3. Lichtenberg FR. The impact of pharmaceutical innovation on premature cancer mortality in Canada, 2000-2011. Int J Health Econ Manag (in press)

4. Harrison C, Kiladjian JJ, Al-Ali HK, Gisslinger H, Waltzman R, Stalbovskaya V, et al. JAK inhibition with ruxolitinib versus best available therapy for myelofibrosis. N Engl J Med 2012;366:787-98.

5. Verstovsek S, Mesa RA, Gotlib J, Levy RS, Gupta V, DiPersio JF, et al. A double-blind, placebo-controlled trial of ruxolitinib for myelofibrosis. N Engl J Med 2012;366:799-807.

6. Martí-Carvajal AJ, Anand V, Solà I. Janus kinase-1 and Janus kinase-2 inhibitors for treating myelofibrosis. Cochrane Database Syst Rev 2015;4:CD010298

7. Reilly JT, McMullin MF, Beer PA, Butt N, Conneally E, Duncombe AS, et al. Use of JAK inhibitors in the management of myelofibrosis: a revision of the British Committee for Standards in Haematology Guidelines for Investigation and Management of Myelofibrosis 2012. Br J Haematol 2014;167:418-20.

8. Vannucchi AM, Barbui T, Cervantes F, Harrison C, Kiladjian JJ, Kroger N, et al. Philadelphia chromosome-negative chronic myeloproliferative neoplasms: ESMO Clinical Practice Guidelines for diagnosis, treatment and follow-up. Ann Oncol 2015; suppl5: v85-99

9. Ho PJ, Marlton P, Tam C, Stevenson W, Ritchie D, Bird R, et al. Practical management of myelofibrosis with ruxolitinib. Intern Med J 2015;45:1221-30.

10. Atkins D, Best D, Briss PA, Eccels M, Falck-Ytter $Y$, Flottrop $S$, et al. Grading quality of evidence and strength of recommendations. BMJ 2004;328:1490-98.

11. NICE appraisal 2016: (accessed on $25^{\text {th }}$ April 2016): https://www.nice.org.uk/guidance/TA386/documents/finalappraisal-determination-document

12. William PL, Webb C. The Delphi technique: a methodological discussion. J Adv Nurs 1994;19:180-186

13. Harrison CN, Vannucchi AM, Kiladjian JJ, Al-Ali HK, Gisslinger H, Knoops L, et al. Long-term findings from COMFORT-III, a phase 3 study of ruxolitinib vs best available therapy for myelofibrosis. Leukemia 2016 (in press)

14. Mesa RA, Kiladjian JJ, Verstovsek S, Al-Ali HK, Gotlib J, Gisslinger H, et al. Comparison of placebo and best available therapy for the treatment of myelofibrosis in the phase 3 COMFORT studies. Haematologica 2014;99:292-8.

15. Mead AJ, Milojkovic D, Knapper S, Garg M, Chacko J, Farquharson M, et al. Response to ruxolitinib in patients with intermediate-1-, intermediate-2-, and high-risk myelofibrosis: results of the UK ROBUST Trial. Br J Haematol 2015;170:29-39.

16. Al-Ali Haifa Kathrin, Griesshammer M, le Coutre P, Waller CF, Liberati AM, Schafausen P, et al. Safety and efficacy of ruxolitinib in an open-label, multicenter, single-arm phase $3 \mathrm{~b}$ expanded-access study in patients with myelofibrosis: a snapshot of 1144 patients in the JUMP trial. Haematologica 2016 (in press)

17. Kiladjian JJ, Giraudier S, Cassinat B. Interferon-alpha for the therapy of myeloproliferative neoplasms: targeting the malignant clone. Leukemia 2016;30:776-81.

18. lanotto JC1, Boyer-Perrard F, Gyan E, Laribi K, Cony-Makhoul P, Demory JL, et al. Efficacy and safety of pegylatedinterferon a-2a in myelofibrosis: a study by the FIM and GEM French cooperative groups. British Journal of Haematology 2013;162:783-91

19. Silver RT, Vandris K, Goldman JJ. Recombinant interferon-a may retard progression of early primary myelofibrosis: a preliminary report. Blood 2011;117:6669-72.

20. Gowin K, Thapaliya P, Samuelsson J, Harrison C, Radia D, Andreasson B, et al. Experience with pegylated Interferon alpha-2a in advanced myeloproliferative neoplasms in an international cohort of 118 patients. Haematologica 2012;97:1570-3.

21. Scherber R, Duecka A, Geyer H, Kosiorek H, Kiladjian JJ, Slots S, et al. Symptoms, risk classification, and spleen size in JAK2 inhibitor-naive myelofibrosis : implications for JAK2 inhibitor treatment. E1345. 21st EHA Meeting 2016. 
22. Vannucchi AM, Kantarjian HM, Kiladjian JJ, Gotllib J, Cervantes F, Mesa RA, et al. A pooled analysis of overall survival in COMFORT-I and COMFORT-II, 2 randomized phase III trials of ruxolitinib for the treatment of myelofibrosis. Haematologica 2015;100:1139-45

23. Verstovsek S, Kantarjian HM, Estrov Z, Cortes JA, Thoams DA, Kadia T, et al. Long-term outcomes of 107 patients with myelofibrosis receiving JAK1/JAK2 inhibitor ruxolitinib: survival advantage in comparison to matched historical controls. Blood 2012;120:1202-9.

24. Passamonti F, Maffioli M, Cervantes F, Vannucchi AM, Morra E, Barbui T, et al. Impact of ruxolitinib on the natural history of primary myelofibrosis: a comparison of the DIPSS and the COMFORT-2 cohorts. Blood 2014;123(12):1833-5.

25. Talpaz M, Paquette R, Afrin L, Hamburg SI, Prchal JT, Jamieson K, et al. Interim analysis of safety and efficacy of ruxolitinib in patients with myelofibrosis and low platelet counts. J Hematol Oncol 2013;6:81.

26. Griesshammer M, Vannucci AM, Le Coutre P, Tavares RS, Al-Ali HK, Raani P, et al. Safety and Efficacy of Ruxolitinib in Patients with Low Platelets Enrolled in a Phase 3b Expanded-Access Study in Myelofibrosis (MF) ASH 2014. Abstract 1859

27. Vannucchi AM, Gisslinger H, Harrison CN, Al-Ali HK, Pungolino E, Kiladjiean J-J, et al. EXPAND: A Phase 1b, OpenLabel, Dose-Finding Study of Ruxolitinib in Patients with Myelofibrosis (MF) and Low Platelet Counts $(50 \times 109 / \mathrm{L}$ to $99 \times 109 /$ L) at Baseline. ASH 2015. Abstract 2817.

28. Galli S, McLornan D, Harrison C. Safety evaluation of ruxolitinib for treating myelofibrosis. Expert Opin Drug Saf 2014;13:967-76.

29. Harrison C, Mesa R, Ross D, Mead A, Keohane C, Gotlib J, et al. Practical management of patients with myelofibrosis receiving ruxolitinib. Expert Rev Hematol 2013;6:511-23.

30. Pieri L, Paoli C, Arena U, Marra F, Mori F, Zucchini M, et al. Long-term follow-up of a phase 2 study of ruxolitinib in patients with splanchnic vein thrombosis associated with myeloproliferative neoplasms, ASH 2015: abstract 2803.

31. Colaizzo D, Amitrano L, Guardascione MA, et al. Outcome of patients with splanchnic venous thrombosis presenting without overt MPN: a role for the JAK2 V617F mutation re-evaluation. Thromb Res 2013;132:e99e104.

32. Ageno W, Riva N, Schulman S, Beyer-Westendorf J, Bang SM, Senzolo M, et al. Long-term Clinical Outcomes of Splanchnic Vein Thrombosis: Results of an International Registry. JAMA Intern Med 2015;175:1474-80.

33. Benjamini O, Jain P, Estrov Z, Kantarjian HM, Verstovsek S. Therapeutic effects of ruxolitinib in patients with myelofibrosis without clinically significant splenomegaly. Blood 2012;120:2768-9.

34. Verstovsek S, Kantanrjian H, Mesa RA, Pardanani AD, Cortes-Franco J, Thomas DA, et al. Safety and efficacy of INCB18424, a JAK1 and JAK2 inhibitor, in myelofibrosis. N Negl J Med 2010;363:1117-27.

35. Isfort S, Kaifie A, Bennemann K, Jost E, Panse J, Bruemmerndorf TH, et al. Comorbidity scales in patients with myeloproliferative neoplasms: which one fits best? Blood 2014;124:1828.

36. EPAR EMA. http://www.ema.europa.eu/ema/index.jsp?curl=pages/medicines/human/medicines/002464/ human_med_001568.jsp\&mid=vvv WC0b01ac058001d124. Accessed on $4^{\text {th }}$ July 2016

37. FDA: ruxolitinib prescribing information. www.accessdata.fda.gov/drugsatfda_docs/label/2011/202192lbl.pdf Accessed on $4^{\text {th }}$ July.

38. Tefferi A, Cervantes F, Mesa R, Passamonti F, Verstovsek S, Vannucchi AM, et al. Revised response criteria for myelofibrosis: International Working Group-Myeloproliferative neoplasms Research and Treatment (IWG-MRT) and European LeukemiaNet (ELN) consensus report. Blood 2013; 122:1395-8.

39. Pardanani A, Tefferi A. Definition and management of ruxolitinib treatment failure in myelofibrosis. Blood Cancer J 2014;4:e268

40. Kroger NM, Deeg JH, Olavarria E, Niederwieser D, Bacigalupo A, Barbui T, et al. Indication and management of allogeneic stem cell transplantation in primary myelofibrosis: a consensus process by EBMT/ELN international working group. Leukemia 2015;29:2126-33.

41. Mehta J, Wang H, Fryzek JP, Iqbal SU, Mesa R. Health resource utilization and cost associated with myeloproliferative neoplasms in a large United States health plan. Leuk Lymphoma 2014;55:2368-74.

42. Ouagari, K, Knight CJ, Mendelson ET. Cost-effectiveness of ruxolitinib versus best-available therapy for medical treatment of myelofibrosis: Canadian societal perspective. American Society of Hematology Meeting 2012; abstract 4255 
43. Loblaw DA, Prestrud AA, Somerfield MR, Oliver TK, Brouwers MC, Nam RK, et al. American Society of Clinical Oncology Clinical Practice Guidelines: formal systematic review-based consensus methodology. J Clin Oncol 2012;30:3136-40.

44. Passamonti F, Cervantes F, Vannucchi AM; Morra E, Rumi E, Pereira A. A dynamic prognostic model to predict survival in primary myelofibrosis: a study by the IWG-MRT (International Working Group for Myeloproliferative Neoplasms Research and Treatment).Blood 2010:115:1703-8.

45. Cervantes F, Dupriez B, Pereira A, Passamonti F, Reilly JT, Morra E, et al. New prognostic scoring system for primary myelofibrosis based on a study of the International Working Group for Myelofibrosis Research and Treatment. Blood 2009;113:2895-601. 